EPJ Web of Conferences 111,01001 (2016)

DOI: 10.1051/epjconf/201611101001

(C) Owned by the authors, published by EDP Sciences, 2016

\title{
The nuclear data, A key component for reactor studies, Overview of AREVA NP needs and applications
}

\author{
Simon Ravaux ${ }^{1}$, Pierre-Marie Demy ${ }^{1}$, and Clément Rechatin ${ }^{2}$ \\ ${ }^{1}$ AREVA NP, PEPDV-F, Tour AREVA - 1 Place Jean Millier, 92084 Paris La Défense Cedex, France \\ ${ }^{2}$ AREVA NP, FE/FL, 10 rue Juliette Recamier, 69456 Lyon Cedex, France
}

\begin{abstract}
The quality of the nuclear data is essential for AREVA NP. Indeed, many AREVA NP activities such as reactor design, safety studies or reactor instrumentation use them as input data. So, the nuclear data can be considered as a key element for AREVA NP. AREVA NP's contribution in the improvement of the nuclear data consists in a joint effort with the CEA. It means a financing and a sharing of information which can give an orientation to the future research axis. The aim of this article is to present the industrial point of view from AREVA NP on the research on nuclear data. Several examples of collaborations with the CEA which have resulted in an improvement of the nuclear data are presented.
\end{abstract}

\section{Short overview of AREVA NP France neutronic activities}

\subsection{Core analysis studies}

AREVA NP performs many core analysis studies. These studies mainly concern core designs, refuelling studies and safety demonstration. They consist in modelling as accurately as possible in a reasonable period the core of a nuclear reactor in steady-state or in transient conditions.

Two core calculation chains are currently available inside AREVA NP France: SCIENCE [1] and ARCADIA [2]. These chains couple many physics fields such as thermal-hydraulic, mechanic and neutronic.

SCIENCE-V2 is the chain which is currently in production inside AREVA NP France. The neutronic part is based on the JEF-2.2 library [3], the spectral code APOLLO2.5 [4] and the core code SMART [1]. This chain has been validated for many applications and is currently used in France for most of the core studies. ARCADIA is a "new" chain which starts its industrial phase. The neutronic part is based on the JEFF-3.1.1 library [5], the spectral code APOLLO2.8 [4] and the core code ARTEMIS [2]. ARCADIA has already been licensed in the U.S [2]. It is currently used in AREVA NP France for internal studies in the perspective of its licensing in France.

\subsection{Other activities}

In addition to the core analysis studies, AREVA NP is also involved in other neutronic activities such as radiation protection studies, fuel assembly conception, residual power calculations... For these activities, many other codes are available inside AREVA NP such as the Monte Carlo code MCNP5[6] and the evolution codes SCALE/ORIGEN-S[7] and ALEPH[8]... 
MCNP5 is considered as a reference code for the criticity and for the neutron propagation. It is used for radiation protection studies, and also to qualify the deterministic codes (including the core calculation chains) through benchmarks and to evaluate uncertainties.

The evolution codes are also essential for AREVA NP. They are used for many applications such as: inventory of used fuel, corium criticity, estimation of delayed neutron or gamma sources, residual power...

\section{Research on nuclear data within AREVA NP France}

All the AREVA NP activities mentioned in the previous chapter make use of nuclear data libraries such as punctual or multigroup cross sections, fission yields, decay data. AREVA NP is involved in the improvement and consequently the research on the nuclear data. This involvement is mainly performed in the frame of the collaboration with the CEA through the so-call "Institut tri-partite" or "I3P".

\subsection{AREVA NP actions inside the I3P}

Inside the I3P, the "direct" research on the nuclear data is mainly performed by the CEA which get a funding from CEA, EDF and AREVA NP. AREVA NP is responsible of the physical validation of its own calculation codes which make use of the nuclear data libraries provided by the CEA. This validation is based on a lot of experimental and power reactor measurements: reactivity, fission rate distribution, depleted pin inventory, boron let-down curve, temperature coefficient, flux map, rod worth... AREVA NP then provides to the CEA a feedback on the basis of the EDF and the worldwide nuclear fleet which can give an orientation to the future research axis on the nuclear data.

\subsection{CEA actions inside the I3P}

The CEA is an important actor inside the European project JEFF. It proposes many new nuclear data evaluations which are then integrated in the new releases of the JEFF library. The new libraries are then transmitted to AREVA NP in binary file readable by AREVA NP codes and more precisely by ARCADIA.

The CEA also designs critical mock-up in experimental reactors. It performs measurements and provides the results to AREVA NP and EDF. These experiments can then be added to the physical validation base of AREVA NP codes.

The last but not least action from the CEA is an expert advice. It can give an efficient support to the industrial needs on the nuclear data.

\section{Example of joint AREVA/EDF/CEA activities}

\subsection{Implementation of the ${ }^{238} \mathrm{U}$ resonant up-scattering}

In the frame of the development of the ARCADIA calculation chain, AREVA NP has noticed some calculation-measurement discrepancies on power reactors. The Reactivity difference (difference on the bore concentration in the moderator) calculated by ARCADIA between the hot zero power (HZP) and the hot full power (HFP) core states, which is also called the HZP/HFP swing, was too low in comparison with the measurements.

In agreement with the CEA, it has been decided to implement the resonant up-scattering model inside the spectral code APOLLO2. The CEA has processed a new multigroup library for APOLLO2 and transmitted it to AREVA NP.

Thanks to these new developments, the discrepancies on the swing have been significantly decreased and the predictability of ARCADIA has been increased. 


\subsection{Feedback on the JEFF-3.1 library}

The CEA delivered to AREVA NP the new application library based on JEFF-3.1 in 2006. AREVA NP quickly observed that, with this new library, the fuel cycle length was slightly under-estimated. This discrepancy was signalled to the CEA.

This problem has been presented by the CEA to the JEFF group. Seven new fission product evaluations were proposed and inserted by the CEA in a new version of the library called JEFF3.1.1[5]. A new application library based on JEFF-3.1.1 was delivered to AREVA NP and allowed to consequently decrease the under-estimation of the cycle length.

\section{Conclusion}

The quality of the neutronic calculations is linked to the quality of the nuclear data libraries. Thus AREVA NP, as a major actor of the nuclear industry, is involved in the research on the nuclear data through the collaboration with the CEA inside the I3P. This collaboration is valuable for both organisms.

\section{References}

1. P. Girieux, proc. of ENC'94 (1994)

2. ARCADIA topical report, http://pbadupws.nrc.gov/docs/ML1015/ML101550189.pdf

3. JEF report 17, https://www.oecd-nea.org/dbdata/nds jefreports/jefreport-17/Table of content.pdf

4. S. Loubiere, R. Sanchez, M. Coste, A. Hebert, Z. Stankovski, C. Van Der Gucht, and I. Zmijarevic, Proc. Int. Conf. Mathematics and Computation, Reactor Physics and Environmental Analysis in Nuclear Applications (1999)

5. JEF report 22, https://www.oecd-nea.org/dbdata/nds jefreports/jefreport-22/nea6807-jeff22.pdf

6. X-5 Monte Carlo Team, LA-UR-03-1987 (2003)

7. S. M. Bowman and I. C. Gauld, ORNL/TM-2010/43 (April 2010)

8. W Haeck, B. Verboomen, SCK CEN-BLG-1003 Rev. 0 (2006) 
